\title{
Le soutien aux travailleurs autonomes : constats issus de deux collectes de données séparées par une période de 10 ans
}

\author{
Sylvie Laferté \\ Professeure en marketing et management \\ École des sciences de l'administration de la TÉLUQ
}

\section{INTRODUCTION}

Dans un article paru dans cette revue même en 2007, nous mentionnons que nous avions observé, sur le terrain comme dans la recherche, que les agences dédiées au soutien des propriétaires dirigeants de PME, des entrepreneurs et des travailleurs autonomes en démarrage ou en opération manquaient de ressources afin de satisfaire les besoins de toutes ces clientèles.

Dans les années 90, le travail autonome a fait l'objet de l'attention des chercheurs et des agences gouvernementales. Leur nombre grandissant, les variations annuelles de ce nombre et la situation économique exigeaient que l'on porte une attention particulière à leurs besoins en encadrement au démarrage et à la gestion de leur travail indépendant, de leur entreprise, si petite fut-elle.

Dans un article paru dans cette revue même en 2007, nous mentionnons que nous avions observé, sur le terrain comme dans la recherche, que les agences dédiées au soutien des propriétaires dirigeants de PME, des entrepreneurs et des travailleurs autonomes en démarrage ou en opération manquaient de ressources afin de satisfaire les besoins de toutes ces clientèles.

L'an dernier, en consultant l'Indice entrepreneurial québécois (IEQ) de 2011, un paragraphe a capté notre intérêt :

«Deux fois plus de démarcheurs ${ }^{1}$ ont sollicité l'aide des services gouvernementaux de soutien à l'entrepreneuriat au Québec $(26,7 \%)$ que dans le reste du Canada (12,6\%), tout comme 1,6 fois plus d'entrepreneurs possédant actuellement une entreprise, que ce soit pour de l'infor- mation, de la formation, de l'aide technique ou de l'aide financière. Par contre, de ceuxci, environ deux fois moins de démarcheurs et propriétaires ont réellement reçu de l'aide au Québec, et ce, malgré deux fois plus de sollicitations en ce sens. Cela entache évidemment leur satisfaction à l'égard des services, puisque près de $20 \%$ des démarcheurs québécois ayant sollicité de l'aide jugent les services comme étant insatisfaisants ou inacceptables, tout comme pour près de $30 \%$ des entrepreneurs québécois, soit un pourcentage d'insatisfaction minimalement deux fois plus élevé que dans le reste du Canada. » (Fondation de l'entrepreneurship $2011:$ 12)

Nous nous sommes immédiatement demandé si les services gouvernementaux d'aide et de soutien à l'entrepreneuriat vivaient encore la situation de manque de ressources que nous avions constaté 10 ans auparavant. Nous nous sommes aussi demandé si les recommandations que nous avions formulées à ce moment étaient encore pertinentes. Cette réflexion nous a amené à refaire une nouvelle collecte de données, avec le même questionnaire, auprès de travailleurs autonomes en opération en 2011, et provenant d'un peu partout sur le territoire québécois.

Dans les pages qui suivent, nous décrirons d'abord les deux collectes de données, celle de 2001 et celle de 2011. Ensuite, nous présenterons et comparerons les résultats de ces deux collectes de données. Avant de conclure, nous reviendrons sur les cinq recommandations que nous avions faites aux organismes de soutien à l'entrepreneuriat suite à la première collecte de données et discuterons de leur pertinence suite à la deuxième collecte de données. 


\section{LES DEUX COLLECTES DE DONNÉES}

257 travailleurs autonomes, dont l'entreprise était en opération, ont répondu à notre questionnaire. Ce questionnaire, format papier, avait été distribué avec un numéro de

L'Autonome, et aux clients des trois organismes précités. Le retour du questionnaire se faisait par la poste. De ces répondants, $70,8 \%$ provenaient du Magazine L'Autonome, 14,8 \% de IDÉE, 7,0 \% du CLD Rimouski Petite Neigette et $7,4 \%$ de la SADC Nicolet Bécancour.

La première collecte de données a été réalisée au printemps 2001 avec la collaboration du défunt Magazine L'Autonome et de trois organismes d'aide aux entreprises, le CLD Rimouski Petite Neigette (cldrn.com), la SADC Nicolet Bécancour (sadcnicoletbecancour.ca), et IDEE - Option Réseau Estrie, maintenant intégré à un autre organisme de développement économique de Sherbrooke. Au final, 257 travailleurs autonomes, dont l'entreprise était en opération, ont répondu à notre questionnaire. Ce questionnaire, format papier, avait été distribué avec un numéro de L'Autonome, et aux clients des trois organismes précités. Le retour du questionnaire se faisait par la poste. De ces répondants, 70,8\% provenaient du Magazine L'Autonome, 14,8\% de IDÉE, 7,0 \% du CLD Rimouski Petite Neigette et 7,4\% de la SADC Nicolet Bécancour.

La deuxième collecte de données, faite en août et septembre 2011, a été réalisé avec la collaboration de deux regroupements de travailleurs autonomes l'AEDQ (www.aedq.org) et AgentSolo (agentsolo.com). La TÉLUQ a aussi été mise à contribution. Le questionnaire, le même qu'en 2001, a été formaté par le biais du site Survey Monkey (surveymonkey.com) et les répondants pouvaient y accéder via un lien hypertexte annoncé d'avance et présent sur les sites Internet des trois organisations déjà mentionnées durant toute la période de collecte. Au final, 110 travailleurs autonomes, dont l'entreprise était en opération, ont répondu à ce questionnaire. De ces 110 répondants, 42,7\% provenaient de la TÉLUQ, 40,0\% de 1'organisme AgentSolo et $17,3 \%$ de l'AEDQ.

\section{COMPARAISON ENTRE LES RÉSULTATS 2001 ET 2011}

Dans cet article, comme dans celui paru en 2007, nous nous attarderons aux résultats concernant les activités de soutien que peuvent offrir ou qu'offrent les services gouvernementaux d'aide aux entreprises, de même qu'aux diverses formes que peuvent prendre les activités de formation dédiées à cette clientèle de travailleurs autonomes. Dans le questionnaire version 2011, nous avons légèrement modifié quelques-uns des énoncés pour les moderniser, sans en changer le sens véritable. Par exemple, un des énoncé débutait par «Site internet chat...», nous l'avons reformulé tout simplement en enlevant le mot chat et les parenthèses du complément d'information présenté dans le tableau 1. Nous aurions pu ajouter des exemples de groupes ou de réseaux internet comme Facebook mais nous avons évalué ces ajouts risqués, pouvant changer le sens que nous donnons à cette activité de soutien possible. Nous avons aussi ajouté quelques exemples plus modernes dans la diffusion de la formation, par exemple la baladodiffusion.

\subsection{La pertinence des services et activités d'aide}

Dans le tableau 1 nous présentons, dans l'ordre de pertinence, les 23 services et activités de soutien que nous avions répertoriés et faits évalués par les répondants en 2001. Dans la colonne de l'extrême droite, nous rapportons l'ordre de pertinence de cette première collecte. Les données sont présentées selon l'ordre de pertinence évalué par les répondants de 2011 (la deuxième colonne de droite).

Nous remarquons d'abord que les quatre premières activités en pertinence sont les mêmes pour les répondants des deux collectes, à 
l'exception des activités 1 et 2 dont l'ordre est inversé. Il semble donc que des activités telles le réseautage, la formation, les regroupements aux fins de lobby et l'aide conseil par des spécialistes aient passé le test du temps. La cinquième activité choisie par les répondants de 2011 est le parrainage ou mentorat, alors que les répondants de 2001 l'avaient placé au onzième rang et avait choisi le maillage comme cinquième activité.

Le mentorat ou parrainage a augmenté sa «cote » de pertinence grâce à l'intensification des efforts et des initiatives de mentorat faits un peu partout au Québec depuis une dizaine d'années.

À notre avis, le mentorat ou parrainage a augmenté sa «cote » de pertinence grâce à l'intensification des efforts et des initiatives de mentorat faits un peu partout au Québec depuis une dizaine d'années; mentionnons les programmes de mentorat de la Fondation de l'Entrepreneurship (entrepreneurship.qc.ca/mentorat-pour-entrepreneurs), Mentorat d'affaires Chaudière-Appalaches (mentoratca.com), et Mentorat d'affaires - Innopole Sherbrooke (sherbrooke-innopole.org/mentorat), pour ne nommer que ceux-là. De la même manière, peut-être parlons-nous moins de réseautage maintenant, ce qui expliquerait en partie sa «décote» par rapport à la collecte de données de 2001.

Nous remarquons ensuite que les dernières activités en pertinence lors de la collecte de 2001 sont sensiblement les mêmes que celles de 2011 à une exception près, le site internet «chat » de 2001 se retrouvait au vingt-troisième et dernier rang alors qu'en 2011, il se retrouve au septième rang. Malgré que $86,4 \%$ des répondants de 2001 possédaient un ordinateur branché à internet, comparativement à 97,3 en 2011 , nous pouvons tous imaginer ici que l'évolution de la technologie (le web 2.0 et le 3.0 qui s'annonce à l'horizon, la tablette électronique et le téléphone intelligent en tête) et de la convivialité de l'utilisation de ces technologies, justifient ce changement dans l'évaluation de la pertinence des solutions sous support internet chez les répondants 2011.

\subsection{L'intérêt pour les formats de formation}

Dans le tableau 2, nous présentons l'intérêt pour les formats de formation selon les répondants de l'échantillon 2001 et de l'échantillon 2011. Ils sont présentés selon la même logique que celle du tableau 1.

La première chose que nous remarquons est que le format de formation à distance est passé du huitième rang en intérêt au premier. Il ressort ici que $42,7 \%$ des répondants proviennent des visiteurs du site internet de la TÉLUQ, qu'il s'agisse d'étudiants ou de visiteurs intéressés par la formule à distance, ce qui peut avoir biaisé les résultats de 2011. Par ailleurs, la formation à distance, tout comme le mentorat et le parrainage, a beaucoup fait parler d'elle dans les dernières années. Au Québec seulement, outre la TÉLUQ, l'Université Laval, l'Université de Sherbrooke, l'ÉNAP, les commissions scolaires et le Cégep à distance offrent tous des cours et des programmes à distance. N'oublions surtout pas iTunes U qui offre des cours ou des parties de cours gratuitement via son diffuseur iTunes de Apple.

\section{La formule « Forum d'experts sur internet » est passée du neuvième rang au deuxième. \\ Encore dans ce cas, il est possible de suggérer que la nouvelle convivialité des outils de communication modernes aura eu un effet sur la perception de la pertinence de ce format de formation.}

La deuxième chose que nous remarquons est que la formule «Forum d'experts sur internet» est passée du neuvième rang au deuxième. Encore dans ce cas, il est possible de suggérer que la nouvelle convivialité des outils de communication modernes aura eu un effet sur la perception de la pertinence de ce format de formation.

Nous pouvons aussi remarquer que les formules individuelles (cours à distance, forum d'experts) ont pris du galon entre 2001 et 2011, mais que les formules en groupe (atelier ou séminaire, colloque) sont presque aussi pertinentes en 2011 qu'en 2001. Dépendamment des sujets et des personnes, on pourrait conclure ici que chaque type de formation (seul ou en groupe) peut répondre à des personnalités et des besoins différents. 


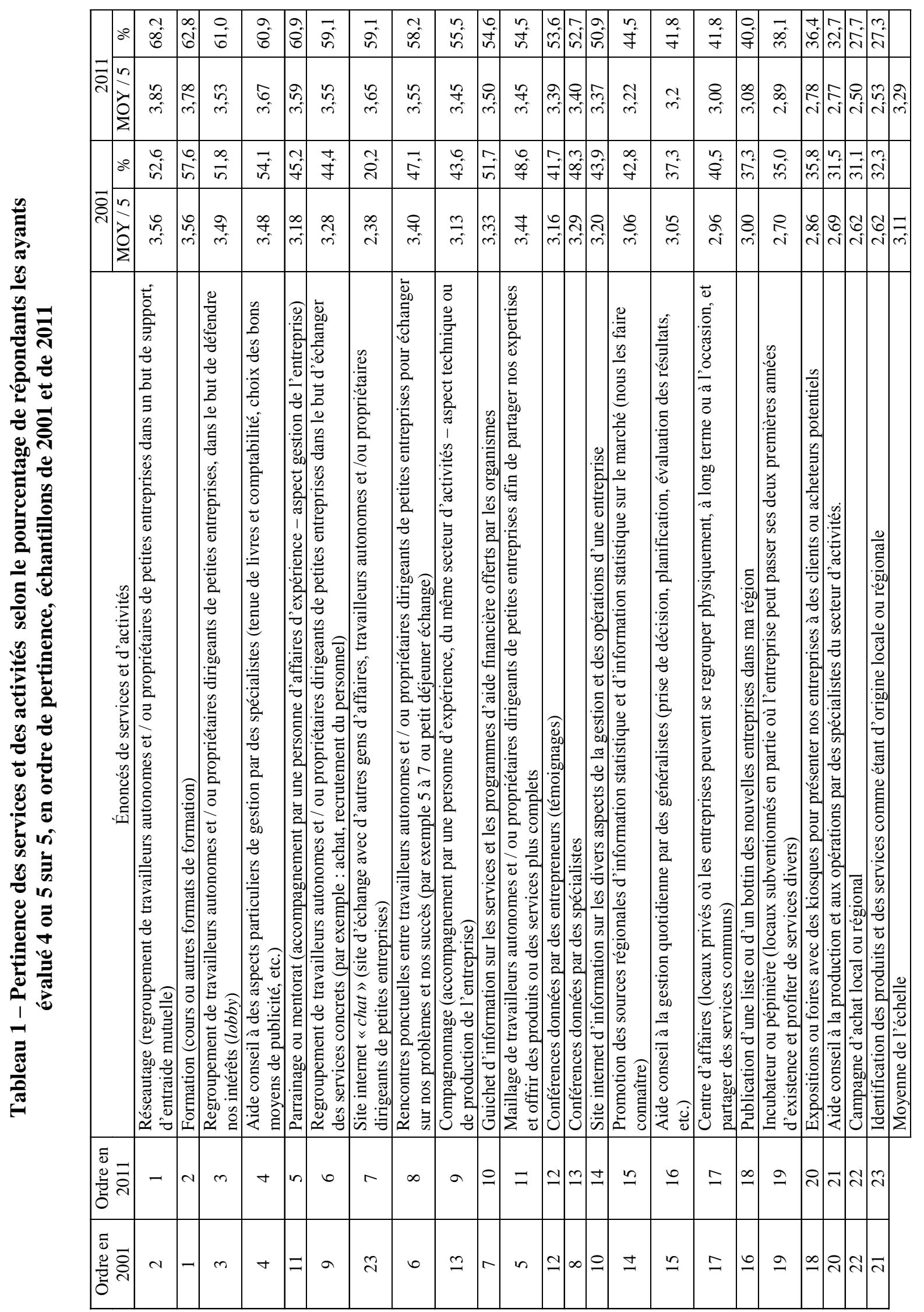




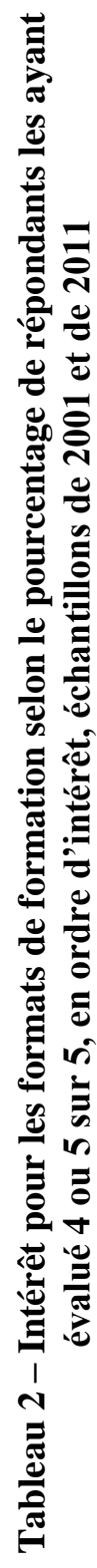

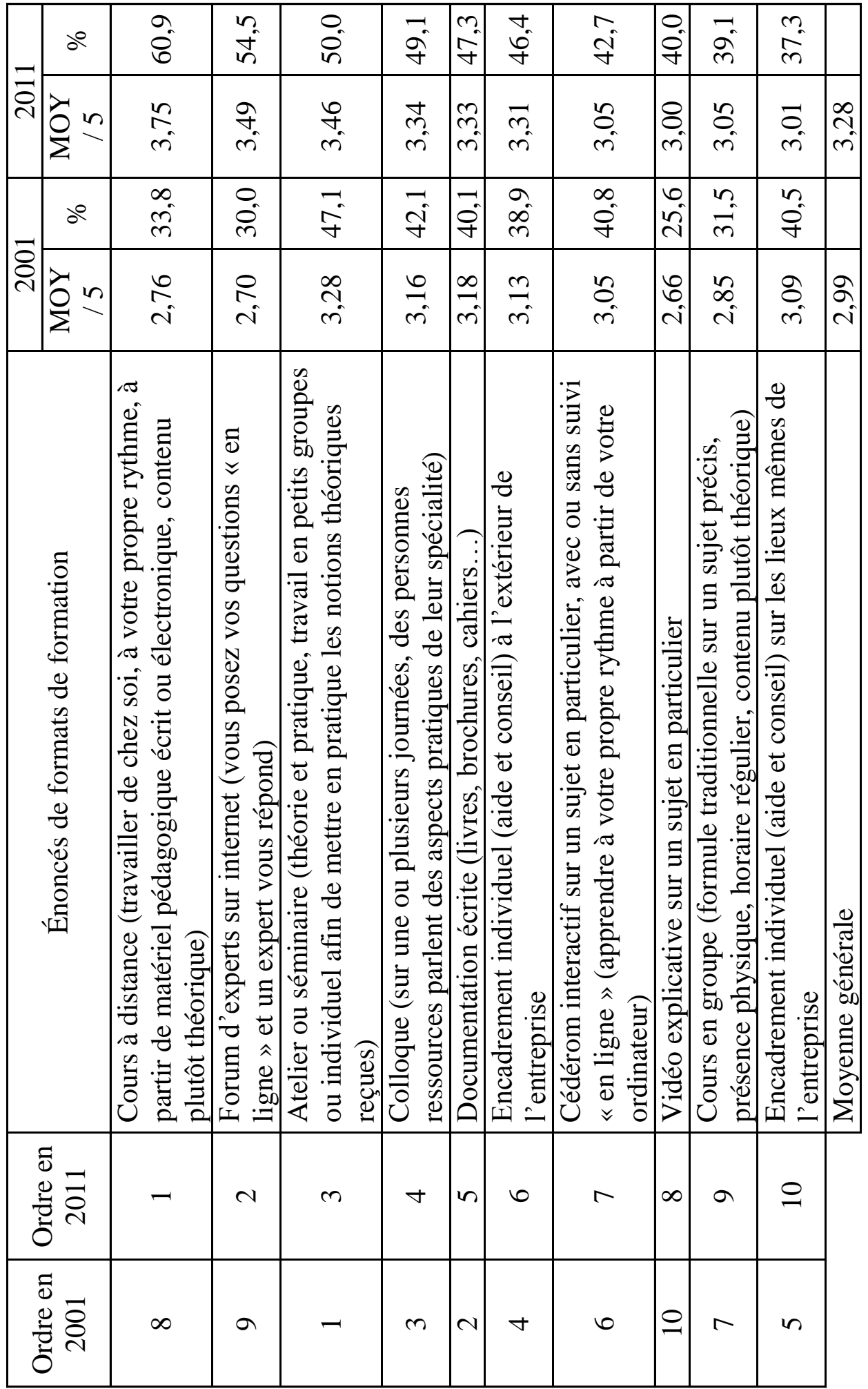




\section{LES RECOMMANDATIONS FAITES À PARTIR DES RÉSULTATS DE 2001 TIENNENT-ILS TOUJOURS LA ROUTE?}

À partir des résultats de la collecte de 2001, nous avions fait cinq recommandations.

1. Lors d'activités où sont invité les travailleurs autonomes, prévoir des périodes d'au moins une heure pendant lesquelles ils pourront échanger librement entre eux ou sur la thématique de l'activité en question;

2. Les activités de formation devraient, prioritairement, utiliser une approche permettant aux participants de comprendre (théorie) et d'appliquer (pratique) les sujets traités, tout en permettant des périodes d'échange;

3. Les activités de soutien offertes par les organismes d'aide aux entreprises devraient être de courte durée ou à durée flexible afin de réduire l'impact du temps passé à participer à l'activité au détriment de l'entreprise;

4. Procéder à une réflexion en profondeur sur les sujets et la forme de l'enseignement à distance afin de répondre aux besoins particuliers des plus petites entreprises;

5. Les organismes d'aide soient les porteurs de ces activités, afin de réduire encore ici le temps passé à participer aux activités au détriment de l'entreprise.

Ces recommandations tiennent encore la route mais nous aimerions y ajouter quelques nuances. $\mathrm{La}$ première recommandation découlait des

\section{CONCLUSION}

Les services d'aide de type réseautage, mentorat, formation, aide conseil et de regroupement semblent aussi pertinents en 2011 qu'en 2001 afin de soutenir les travailleurs autonomes dans la gestion de leur entreprise.

L'étude comparative sur une période de 10 ans sur le soutien des travailleurs débouche sur des constats intéressants : 1) les services d'aide de type réseautage, mentorat, formation, aide conseil et de réponses aux activités et services les plus pertinents qui touchaient principalement celles en groupe, celles où les travailleurs autonomes pouvaient échanger entre eux. Même si l'ordre de pertinence a quelque peu changé, cette recommandation demeure valide. La deuxième recommandation découlait du fait que la formule d'atelier ou de séminaire était la première en intérêt. Même si elle est deuxième selon la collecte de 2011, elle demeure toujours valide puisque la pratique est déterminante dans la compréhension de la théorie - pensons à la comptabilité et au processus de gestion par exemple. Le temps étant toujours un problème pour les travailleurs autonomes, les entrepreneurs et pour la grande majorité de la population, compte tenu que les travailleurs autonomes paient doublement le prix des activités auxquelles ils participent (temps dédié à l'activité au détriment de l'entreprise), nous croyons que les recommandations 3 et 5 tiennent encore la route.

La quatrième recommandation portait sur la formation à distance. Aujourd'hui, selon les résultats de la collecte de données 2011, nous aurions tendance à lui donner une priorité plus élevée dans la liste de nos recommandations. Il faut cependant tenir compte du biais provenant $\mathrm{du}$ fait que près de $43 \%$ de l'échantillon 2011 provient des personnes fréquentant le portail de la TÉLUQ, donc déjà intéressées sinon impliquées dans la formule à distance.

regroupement semblent aussi pertinents en 2011 qu'en 2001 afin de soutenir les travailleurs autonomes dans la gestion de leur entreprise, si petite soit-elle; et 2) que les technologies de communication et de diffusion de la formation étant de plus en plus conviviales pour les utilisateurs et les diffuseurs, leur utilisation devient un incontournable afin de soutenir ces travailleurs autonomes.

Quoiqu'il en soit, la formation est toujours en haut de la liste des activités ou services que peuvent 
offrir les organismes d'aide aux travailleurs autonomes, qu'elle se fasse à distance ou en présentiel. De plus, si l'on retient nos cinq recommandations, la formation devra être de courte durée et à durée flexible, permettra les échanges entre les participants, inclura des périodes d'apprentissage théorique et d'application pratique et sera organisée et soutenue par les organismes d'aide aux travailleurs autonomes. Deux objectifs seraient alors atteints : un soutien mieux ciblé auprès des travailleurs autonomes dans la gestion de leur entreprise et une affectation plus efficace de leurs ressources pour les organismes de développement économique. Cette conclusion reprend, l'esprit sinon la lettre, de nos conclusions tirées des résultats de 2001.

\section{La formation devra être de courte durée et à durée flexible, permettra les échanges entre les participants, inclura des périodes d'apprentissage théorique et d'application pratique et sera organisée et soutenue par les organismes d'aide aux travailleurs autonomes.}

\section{BIBLIOGRAPHIE}

Fondation de l'entrepreneurship (2011). Culture entrepreneuriale au Québec: nos entrepreneurs prennent-ils racine? Québec: Fondation de l'entrepreneurship.

Laferté, S. (2005). Le soutien aux travailleurs autonomes en exercice : proposition aux organismes de développement économique, thèse de doctorat, Université du Québec à Trois-Rivières.

Laferté, S. (2007). Le soutien aux travailleurs autonomes en exercice : constats issus de la recherche et réflexions nourries par le terrain, Organisation \& Territoires, vol. $16 \mathrm{n}^{\text {os }} 2$ et 3, été-automne 2007.

\section{NOTES}

${ }^{1}$ Personnes faisant des démarches en vue de créer une entreprise. 


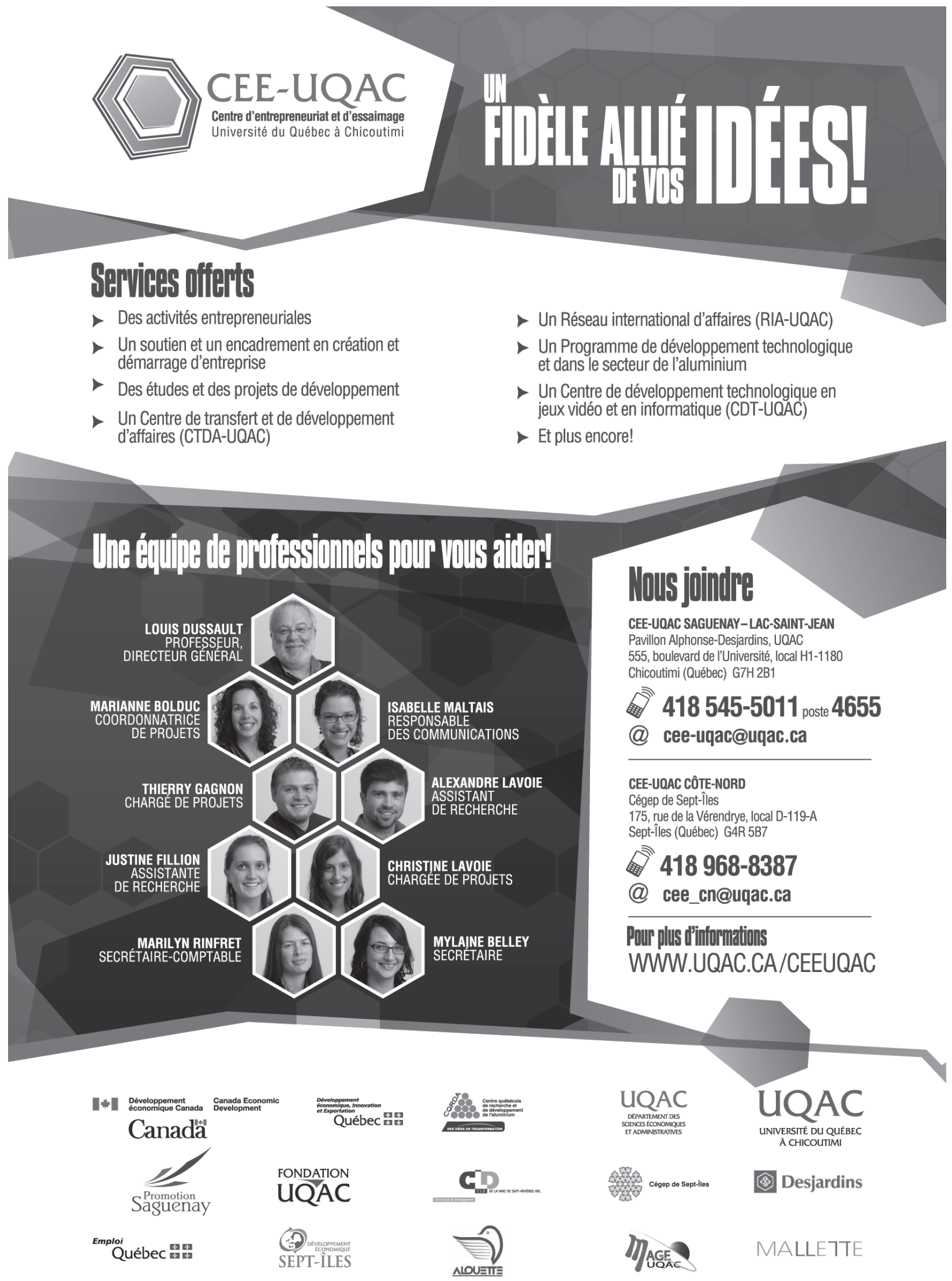

